\title{
The COVID-19 pandemic requires a unified global response
}

\author{
Sebastiano Filetti ${ }^{1}$ \\ Published online: 6 April 2020 \\ (c) Springer Science+Business Media, LLC, part of Springer Nature 2020
}

The ongoing spread of the COVID-19 pandemic is having profound effects on our private and professional lives, the world economy, and the social organization of the communities we live in. This moment in history is so dramatic as to seem incomprehensible to some, to others a scene straight out of a disaster movie. Those of us who have devoted our lives to understanding and combatting illness know that science is the key to halting this disaster, but we are also aware of the fact that the shape of the world we live will be strikingly altered by this pandemic.

Today, our tasks are embodied by the roles we have in society: to be present, to be committed, and to participate wholeheartedly in the fight against the coronavirus outbreak. Foremost in my mind, therefore, is a sense of gratitude and pride when I think of the health professionals on the front lines of the war against this new viral disease, dedicated doctors, nurses, and other paramedicals-our friends and colleagues-working tirelessly and risking their lives to save patients and find the answers we need.

The world of endocrinology and metabolism is also immersed in the pandemic, in hospital wards and outpatient clinics, where endocrinologists are supporting high-risk COVID-19 patients, elderly individuals with diabetes or endocrine cancers, and providing ongoing care for the vulnerable patient population with chronic endocrine diseases. With this in mind, I would like to personally thank Prof. Andrea Giustina, former editor-in-chief of Endocrine and current President of the European Society of Endocrinology, for asking us to publish the Society's statement on the COVID-19 pandemic. The statement appears in this issue of Endocrine [1], and we are convinced it will be of service to our community of readers. It offers a broad range of expert advice on counseling, monitoring, and treating patients with diabetes and endocrine disorders during this difficult time, regardless of whether or not they have COVID-19. I am sure that it will prove to be a valuable resource for all of you.

In the meantime, all of us here at Endocrine are committed to remaining fully operational, ensuring timely completion of constructive, objective peer reviews, and minimizing publication delays.

We encourage COVID-19-related research submissions regarding research on the relationship between COVID-19 and endocrine disorders as well as on the experience of diabetic and endocrine patients subject to lockdown and social distancing guidelines (https://www.springer.com/ journal/12020/updates/17856320). I would like to take this opportunity to thank the journal's associate editors, who continue to carry out their responsibilities with dedication and punctuality despite significant demands from their own institutions and daily routines. And our efforts would be in vain without the support of the dedicated staff at Springer Nature and the experience and ability of Lisa Hussey, the journal's Publishing Editor.

As always, they are attentive to our requests and quick to find solutions, even in this critical period.

With the sincere hope that this crisis will soon be over, I remain.

\section{Reference}

1. M. Puig, M. Marazuela, A. Giustina. COVID-19 and endocrine diseases. A statement from the European Society of Endocrinology. Endocrine 68, (2020). https://doi.org/10.1007/s12020-020-02294-5
Sebastiano Filetti

editor.endocrine@gmail.com

1 Sapienza University of Rome, Rome, Italy 